\title{
The hypercortex and the context of the convergence of art with info-cognotechnologies
}

\author{
Keywords \\ Digital arts, Hypercortex, Relationship between art-science-technology, Telematic art, Info-cognotechnology.
}

The work presented here brings a reflection originated from the group Laboratório de Poéticas Fronteiriças (http://labfront.tk), registered at Brazilian Council for Scientific and Technological Development directory and certified by the State University of Minas Gerais. Here we show a snippet, based on some of our bibliographical research, that aims to bring together a specific aspect of digital arts known as "telematic art". Besides being expressed through telematics systems, we understand telematic artworks in regards to their connections to infotechnologies and cognotechnologies in the context of the scientific and technological convergence we are currently navigating through. Infotechnologies deal directly with the moment in human history in which we have entered since half of the 20th century. An era based on information, where multiple technologies allow access to a large amount of data and knowledge, enabling for an even further development in research on various areas. It is a development from the usual way in which we access information, making for a more direct access to a multitude of means thanks to the implementation of digitally attained and sustained databases, research methods, and communication. Cognotechnologies, on the other hand, are the developments that allow for a cognitive connection. They artificially recreate how the human brain works, through neuroscientific discoveries and relating with the way our mind works, presenting itself as a disruptive technology enabling the extrapolation of traditional infotechnological interactions between humans and machines, enabling a sort of neural network to be developed where, thanks to the use of diverse specific technologies we can build a hyperconnection amongst people, mediated by the machine. Having said that, we bring to the discussion the idea of the hyper-cortex. It is anchored in the relationship between the idea of "shared global intelligence" and the extrapolation of humans' brain-pan. The info-cognotechnological developments create a transformative and mediative individual cognitive processes hypercortex, changing the modus operandi of social relationships. This way, by understanding the biological function of the human cortex, which is directly connected to the hyper-cortex, we are able to realize the possibility of expanding its ability with the help of technological methods. Furthermore, these methods make it so that such human consciousness expansion transcends beyond its physical dimension, allowing for a linkage on the human-machine and machinemachine processes. This idea, here vastly supported by the reflections made by Roy Ascott (2003) as well as Pierre Levy (2017), deals directly with the possibilities of expansion of the human neocortex. In our work, we analyze digital artworks in which the info-cognotechnological dimension is poetically explored in search of a scientific, technological, and artistic convergence. With all this, we are able to demonstrate how different artworks end up conceptually - or in a theoreticalpractical way - implementing what humanity experiences physically. 